\title{
A RELAÇÃO DO ESTRESSE EM ENFERMEIROS NO TRABALHO HOSPITALAR
}

THE RELATIONSHIP OF STRESS IN NURSES IN HOSPITAL WORK

Ciély Ferreira Goedert

Pós-Graduação em Gestão de Pessoas com Coaching pelo Instituto Doll, bacharel em Psicologia pela Faculdade Sant'Ana. 


\section{RESUMO}

Este trabalho visa realizar um levantamento sobre a predominância dos sintomas físicos e psicológicos de estresse dos profissionais de enfermagem que atuam em hospitais da cidade de Ponta Grossa/PR. Como método para o alcance do objetivo foi confeccionado e aplicado um questionário sócio demográfico e também aplicado o instrumento ISE - Inventário Sintomas de Estresse construído por Ana Maria T. Benevides Pereira \& Bernardo Moreno Jiménez (2000), a ferramenta foi validada estatisticamente alcançando um alfa de Crombach de 0,9236. O ISE é constituído por escala de Likert de 5 pontos, indo de 0 como "nunca" a 4 como "assiduamente". A população escolhida foi de 103 profissionais de enfermagem na qual foram questionados uma amostra de 76 enfermeiros com o grau de confiança de $95 \%$ sobre a população. Em relação aos resultados alcançados na pesquisa pode-se afirmar que atualmente os enfermeiros apresentam predominância de sintomas físicos em média de 1,99 e psicológicos em média 1,95, considerado um nível médio de estresse. O resultado é extremamente grave, pois dentro dessa média provavelmente existam pessoas com nível baixo e outras com nível elevado, isto tendencia uma estabilidade ao longo do tempo para um nível alto de estresse.

Palavras-chave: Estresse, enfermagem, ambiente hospitalar

\section{ABSTRACT}

This work aims to indicate the level physical and psychological symptoms of stress of nurses working in hospitals in the city of Ponta Grossa / PR. As method to reach the goal was built and applied a demographic questionnaire and also was used the ISE instrument - Stress Symptoms Inventory constructed by Ana Maria T. Pereira Benevides \& Bernardo Moreno Jiménez (2000), the tool was validated statistically with Cronbach alpha of 0.9236. The ISE is composed of Likert scale of 5 points, ranging from 0 as "never" to 4 as "assiduously". For population chosen of 103 nurses, the research was conducted as 76 nurses for achieve with the confidence level of $95 \%$ of the population. Regarding the results achieved in research may be stated that currently the nurses (as) have an average of 1.99 for physical symptoms and 1.95 to average psychological symptoms, considered a medium level of stress. The result is extremely 


\section{A RELAÇÃO DO ESTRESSE EM ENFERMEIROS NO TRABALHO HOSPITALAR}

serious, because within this mean there probably are people with low and those with high level, this number should lead, over time, stability for level high.

Keywords: Stress symptoms, nursing, work 


\section{INTRODUÇÃO}

O estresse está presente desde a antiguidade, quando o homem lutava pela sua sobrevivência buscando seu alimento, enfrentando guerras entre tribos, sobrevivendo às catástrofes do tempo, lutando a cada dia pela conquista de um território entre várias situações.

Atualmente as situações vivenciadas pelo homem também estão ligadas a fatores que podem favorecer ou interferir inclusive na performance do profissional de enfermagem no seu ambiente de trabalho. Entretanto no Brasil ainda existe insuficiência de estudos para delimitar os fatores que levam os profissionais de enfermagem para o alto nível de estresse. Evidenciase que a atividade do profissional de enfermagem pela sua própria condição de trabalho o expõe a determinados riscos que podem levar ao desenvolvimento por estresse (SILVA; SANTOS; NASCIMENTO, 2016).

O estresse se apresenta em muitas áreas de atuação profissional, principalmente em profissão que têm como missão servir outros indivíduos alguns estudos destacam a profissão de professor, médico, enfermeiro entre outros. Este estudo apresenta um enfoque sobre o estresse na equipe de enfermagem que tem exibido em pesquisas como sendo considerado o tema importante para a sociedade.

Este estresse ocupacional para a equipe de enfermagem tornou-se parte do cotidiano da profissão, por sua diversidade de tarefas e o compromisso em oferecer assistência com maior qualidade. O desgaste emocional com o qual a equipe de enfermagem se depara, ao assumir as atribuições relativas ao cargo, a exposição pode ser altamente marcante, devido à diversidade de situações que um ambiente hospitalar oferece aos enfermeiros.

O levantamento do nível de estresse e a identificação dos estressores no ambiente hospitalar serão de grande importância para o desenvolvimento e aprimoramento de uma intervenção psicológica mais adequada, dentro deste espaço da pesquisa que hoje ainda se apresenta como um campo a ser explorado. Permite ainda, a constatação da teoria disponível com a realidade estudada, tornando-se uma fonte de informação para outras pesquisas, o que permite aproximar o ensino da academia com as necessidades encontradas nos atuais ambientes de trabalho. Como bem escreve os autores Ueno LGS, Bobroff MCC, Martins JT (2016) “O estado prolongado de preocupação, alerta e ansiedade caracteriza-se em uma intensa carga de estresse transformando-se num oponente a ser vencido. Se os fatores estressantes persistirem, em frequência ou intensidade, há quebra da resistência do indivíduo e ele passa à fase de quase 
exaustão levando a possíveis doenças como, por exemplo, a depressão, interferindo tanto na vida pessoal, quanto na atividade laboral".

No contexto descrito acima a pesquisa se norteará em busca de responder a seguinte questão: Qual a predominância dos sintomas físicos e psicológicos de estresse presentes no enfermeiro atuante nos hospitais na cidade de Ponta Grossa/PR?

\section{METODOLOGIA}

O ponto inicial de uma pesquisa está em um problema que faça jus a ser estudado, com o objetivo de propor uma solução. A pesquisa começa quando há uma vontade do conhecimento, de ordem intelectual ou quando há este desejo, mas existe uma necessidade de fazer algo de uma maneira mais eficiente e eficaz.

No caso desta pesquisa, foi utilizado o método dedutivo que pressupõe que só a razão é capaz de chegar a um conhecimento confiável. A presente pesquisa foi realizada no ano de 2017, contou com a participação de 7 (sete) hospitais, os quais não permitiram a identificação de suas razões sociais.

Do ponto de vista da natureza este estudo se classifica como sendo uma pesquisa básica, bibliográfica pelo fato de ser realizado um levantamento bibliográfico inicialmente, feito uma busca pela literatura e materiais já publicados constituídos principalmente de artigos de periódicos, livros ou outro material atualmente disponibilizado pela internet.

Para a pesquisa com uma população de 76 (setenta e seis) enfermeiros, foi aplicado um questionários sócio demográfico composto por 12 (doze) perguntas, tratando de gênero, faixa etária, instituição a que pertence endereço, estado civil, quantidade de filhos, tipo de residência, ano de formação, tempo de experiência na área, sentimento na atuação da profissão, turno de trabalho e quantidade de instituições em que atua.

Também utilizado o instrumento para a coleta de dados, com o objetivo de identificar os sintomas de estresse; questionário ISE (Inventário de Sintomas de Estresse) elaborado e autorizado para utilização nesta pesquisa por Ana Maria T. Benevides Pereira \& Bernardo Moreno Jiménez. 


\section{RESULTADOS}

O gênero mais predominante entre os 76 enfermeiros é do sexo feminino, alcançando um índice de $79 \%$ em relação ao total da amostra. Assim o sexo masculino aparece levemente com apenas $21 \%$ em relação ao total.

Com relação à faixa etária dos profissionais de enfermagem na região, que apesar da diversidade existe uma maior concentração com idades de 20 a 30 anos alcançando um percentual de $57 \%$ em relação ao total de 76 pesquisados, em segundo lugar pertencem a uma faixa etária entre 31 a 40 anos com 30\%, na sequencia encontramos $12 \%$ na faixa etária de 41 a 50 anos e menos de $1 \%$ em relação ao total foi identificado acima de 51 anos.

O resultado deste levantamento indica que $61 \%$ dos enfermeiros atuantes nos hospitais de Ponta Grossa são de origem da cidade, por outro lado podemos dizer que existe um grande número de profissionais que pertencem a outras regiões, pois alcança $39 \%$ do total pesquisado. Indicativo do tipo de residência dos enfermeiros.

Maior parte dos enfermeiros foram formados menos de 5 anos, o que representa um número de pessoas com pouca experiência profissional, esse grupo representa $80 \%$ em relação ao número de enfermeiros que participaram da pesquisa. Outros profissionais realizaram sua formação entre os anos de 1998 e 2003 e representam 13\% dos pesquisados..

Por fim, outros 3\% dos pesquisados estão na escala de formação de 1980 à 1985, outros 4\% na escala de 1986 à 1991 e não tivemos nenhum formando entre 1992 à 1997.

Uma diversidade sobre o tempo de experiência dos enfermeiros foi identificado conforme a figura 11, a maior percentual está entre 2 a 3 anos de experiência com $30 \%$ do total pesquisado, posteriormente $25 \%$ possuem menos de 1 ano de experiência, $20 \%$ estão na escala de 4 a 5 anos, $13 \%$ possui de 6 a 7 anos e 12\% são mais experientes de todos com mais de 8 anos de atuação na área de enfermagem.

Quando questionado sobre o sentimento sobre a atuação do profissional em relação sua profissão tivemos uma grande satisfação conforme mostra a figura 12, a explicitação predomina com muito bem alcançando $62 \%$ dos entrevistados, resposta com sentimento de sentir-se bem ficou em 33\% em relação ao total, com $4 \%$ identificamos uma dúvida sobre a satisfação marcando a opção mais ou menos, menos de $1 \%$ respondeu sentir-se mal na atuação e nenhum profissional sente muito mal em atuar na sua profissão.

Para maior entendimento foi codificada as variáveis por M - Manhã, T - Tarde, N Noturno, MT - Manha e Tarde, MTN - Manha, Tarde e Noite, MN - Manha e Noite e TN - 
Tarde e Noite. Foi identificado que grande parte não possui um período fixo de trabalho, com isso percebe-se que no período manha existem 52 enfermeiros da amostra, 46 atuantes à tarde e 28 dos enfermeiros atuam no período noturno. Dos enfermeiros que atuam em mais de uma instituição o maior número está atuando no período de manha e tarde com $28 \%$ do total.

\subsection{Indicativos sobre a predominância dos sintomas físicos}

Para uma verificação mais refinada sobre os dados adquiridos através do instrumento ISE, foi identificado como fundamental demonstrar através da tabela 1, alguns números levantados estatisticamente sobre os sintomas físicos. Primeiramente podemos destacar a predominância dos sintomas físicos como médio pelo resultado alcançado de $49 \%$ sobre as respostas coletadas e percebe-se que o nível baixo e alto se encontra no mesmo patamar com $25 \%$ para nível alto e $26 \%$ para o baixo.

\subsection{Indicativos sobre a predominância dos sintomas psicológicos}

$\mathrm{Na}$ mesma direção utilizada sobre a ilustração dos sintomas físicos é significante executar sobre os sintomas psicológicos. O baixo nível de sintomas psicológicos foi encontrado com a marca de $38 \%$ em relação ao total pesquisado, mas um grande número se concentra no nível alto de sintomas psicológicos com 33\%. Um índice considerável sobre o médio nível de sintomas psicológicos com $29 \%$ concluindo que existe uma disparidade sobre esses sintomas.

\subsection{Resultado geral sobre sintomas físico e psicológico de estresse}

Para finalização da análise dos dados identificamos através de um levantamento estatístico considerado pelo instrumento como forma de explicitar o resultado alcançado, o qual demonstrou que a pontuação é limítrofe para médio no caso de 1,99 para o físico e 1,95 para o psicológico sobre a média levantada.

No levantamento da mediana sobre os dados coletados observamos uma igualdade sobre a predominância dos sintomas, pois ambos alcançaram o valor 2. Diferentemente da moda que alcançou o valor 2 para o físico e apenas 1 para o psicológico.

Percebemos que ficou um desvio padrão mais leve para os sintomas físicos com 0,72 e não muito maior o desvio para os sintomas psicológicos foi de 0,84 sobre os dados tabulados 


\section{CONSIDERAÇÕES FINAIS}

Este trabalho de conclusão de curso se propôs a realizar um levantamento sobre a predominância de sintomas físicos e psicológicos de estresse nos enfermeiros dos hospitais pertencentes da cidade de Ponta Grossa/PR.

Através da aplicação dos instrumentos escolhidos podemos concluir que existem alguns aspectos relevantes identificados na execução da pesquisa que valem a pena destacar, são eles:

- Em relação aos dados sócios demográficos podemos dizer que existe a possibilidade de interferir o resultado final de sintomas devido algumas variáveis como: experiência como profissional de enfermagem; Idade; quantidade de instituições na qual atua e turno de trabalho que fazem.

- Sobre o levantamento dos sintomas físicos de estresse conclui-se que existe a tendência de alcançar o nível alto nos próximos anos, principalmente quando os profissionais alcançarem maior tempo de atuação, pois como explicitado, atualmente já se encontra no nível médio;

- Sobre a predominância dos sintomas psicológicos foi identificado um nível médio com grande possibilidade de chegar ao um nível alto devido ao resultado alcançado.

Por fim como forma de consolidar a conclusão podemos discorrer que através dos resultados alcançados a amostra possui indícios de estresse nível médio para sintomatologia física e psicológica o que é extremamente grave, pois dentro dessa média provavelmente existam pessoas com nível baixo e outras com nível elevado.

Com base neste cenário podemos afirmar que o número de pessoas com alto nível de sintomas de estresse tende a aumentar, pois os dados sócios demográficos deverão, ao longo do tempo, se estabilizar e assim a amostra ficará mais equilibrada.

\section{CONCLUSÃO}

O estresse na organização hospitalar pode ser considerado como uma fonte de pressão física e psicológica, assim como em outros âmbitos de trabalho como fonte de renda financeira. Pode ser relacionado ao grupo de trabalho, formato da organização ou do histórico emocional de cada profissional que se identifica com a área de enfermagem hospitalar.

É válido mencionar, que o estresse é um fenômeno subjetivo, relacionado a percepção de cada indivíduo; sendo os fatores potencializadores do estresse quase sempre não são os mesmos para todas as pessoas. 
No contexto do ambiente hospitalar, os profissionais inseridos neste grupo, muitas vezes podem ser vistos como profissionais que possuem uma auto exigência, sonhadores com o mundo ideal, pessoas dedicadas e ao mesmo tempo com muitas dificuldades de blindar seu emocional mediante situações de enfermidade. Onde são vistos pela capacidade ou não de lidar com dificuldades, tristezas e desequilíbrios emocionais nos casos de doenças ou situações inesperados que levam pacientes no contexto hospitalar.

Outro ponto a ser avaliado, são as manifestações comuns nesses colaboradores, por apresentar irritabilidade, ansiedade, sem interesse pela vida ou outra situação de normalidade no contexto familiar ou profissional, pode reduzir seu comprometimento com o trabalho, deixando de lado seu compromisso com resultados da organização.

Pelo fato do trabalho ser exaustivo, fisicamente e emocionalmente, exige-se uma energia adicional para evitar um esgotamento emocional do profissional de enfermagem. Sugere-se uma flexibilidade de horários com redução de jornada de trabalho; assim pode-se ter um ganho em produtividade e atenção reduzindo a carga emocional afastando-o mais horas de suas atividades para descanso mental.

\section{REFERÊNCIAS}

SILVA, Damiana; SANTOS, Nadson Ricly \& NASCIMENTO, Luzia Kelly Alves da Silva . Fatores que influenciam o Estresse Ocupacional na Enfermagem. Revista Cultural e Científica do UNIFACEX [online], vol.14, n. 2, 2006. ISSN: 2237 - 8685. Disponível em: https://bit.ly/2I88Qbi. Acesso em: 16 fev. 2020

Ueno LGS,;Bobroff MCC \& Martins JT. Estresse Ocupacional: Estressores Referidos pela Equipe de Enfermagem. Revista de Enfermagem UFPE [online], vol. L, 2017. Disponível em: https://bit.ly/2VwyWgo. Acesso em: 16 fev. 2020

BIANCHI, Estela Regina Ferraz. Estudo exploratório sobre suspensão de cirurgia: a comunicação da suspensão e a reação relatada pelo paciente frente ao fato. 1983, (dissertação). Escola de Enfermagem, Universidade de São Paulo, São Paulo, 1983.

BIANCHI, Estela Regina Ferraz. Escala Bianchi de Stress. Revista de Enfermagem da Universidade de São Paulo [online], vol.43, n.spe, pp. 1055-1062, 2009. Disponível em: https://bit.ly/2I3f50n. Acesso em: 20 fev. 2020. 